\title{
Imaging Biomarkers and Their Impact on Therapeutic Decision-Making in the Management of Neovascular Age-Related Macular Degeneration
}

\author{
David T. Wong $^{\mathrm{a}}$ Alan R. Berger ${ }^{\mathrm{a}}$ Serge Bourgault ${ }^{\mathrm{b}}$ John Chen ${ }^{\mathrm{c}}$ \\ Kevin Colleaux $^{d}$ Alan F. Cruess ${ }^{e}$ Ravi I. Dookeran ${ }^{f}$ Danny Gauthier ${ }^{g}$ \\ Bernard Hurley $^{\text {h }}$ Michael A. Kapustac ${ }^{c}$ Peter J. Kertes ${ }^{a}$ Cynthia X. Qian ${ }^{g}$ \\ Arif Samad $^{\mathrm{e}}$ Thomas Sheidow $^{i}$ James H. Whelan ${ }^{j}$

\begin{abstract}
${ }^{a}$ Department of Ophthalmology and Vision Sciences, University of Toronto, Toronto, ON, Canada; ${ }^{b}$ Department of Ophthalmology, Université Laval, Québec, QC, Canada; ' Department of Ophthalmology, McGill University, Montréal, QC, Canada; 'D Department of Ophthalmology, University of Saskatchewan, Saskatoon, SK, Canada; e Department of Ophthalmology and Visual Sciences, Dalhousie University, Halifax, NS, Canada; ${ }^{\mathrm{f}}$ Department of Ophthalmology, University of Manitoba, Winnipeg, MB, Canada; ${ }^{9}$ Department of Ophthalmology, Université de

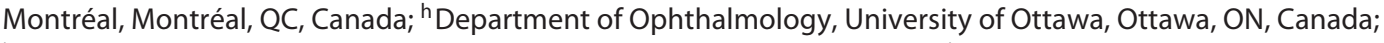

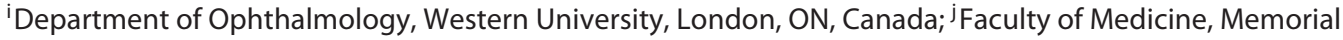
University, St. John's, NL, Canada
\end{abstract}

\section{Keywords}

Neovascular age-related macular degeneration .

Biomarkers · Fluid · Intraretinal · Subretinal

\begin{abstract}
These recommendations, produced by a group of Canadian retina experts, have been developed to assist both retina specialists and general ophthalmologists in the management of vision-threatening neovascular age-related macular degeneration (nAMD). The recommendations are based on published evidence as well as collective experience and expertise in routine clinical practice. We provide an update on practice principles for optimal patient care, focusing on
\end{abstract}

identified imaging biomarkers, in particular retinal fluid, as well as current and emerging therapeutic approaches. Algorithms for delivering high-quality care and improving longterm patient outcomes are provided, with an emphasis on timely and appropriate treatment to preserve and maintain vision. In the context of $n A M D$, increasing macular fluid or leakage on fluorescein angiography (FA) may indicate disease activity regardless of its location. Early elimination of intraretinal fluid (IRF) is of particular relevance as it is a prognostic indicator of worse visual outcomes. Robust referral pathways for second opinion and peer-to-peer consultations must be in place for cases not responding to intravitreal anti-vascular endothelial growth factor (anti-VEGF) therapy. karger@karger.com

(c) 2021 S. Karger AG, Basel

www.karger.com/oph

Karger ${ }^{\prime \prime}=$
Correspondence to:

David T. Wong, david.wong@unityhealth.to 


\section{Introduction}

Neovascular age-related macular degeneration (nAMD) is characterized by the development of choroidal neovascular membranes. This growth of abnormal blood vessels causes an accumulation of fluid and blood in the intraretinal and subretinal spaces, which can have a detrimental impact on visual acuity (VA) if left untreated [1]. Over the past decade, intravitreal anti-vascular endothelial growth factor (anti-VEGF) therapy has been established as the standard of care for the treatment of nAMD by controlling the exudative process and improving visual outcomes.

The landmark MARINA and ANCHOR studies involving fixed monthly injections of ranibizumab set a high standard for VA results in nAMD treatment $[2,3]$. Subsequently, other molecules (aflibercept and brolucizumab) $[4,5]$ and treatment approaches [6-11] have been designed, in an attempt to obtain the same favorable results, while treating with a reduced number of intravitreal injections. Treat-and-extend (T\&E) [6-8] and asneeded [9-11] regimens use the presence of fluid on optical coherence tomography (OCT) as a key biomarker of nAMD activity, driving decisions regarding the need for retreatment.

This paper examines the latest evidence regarding imaging biomarkers, specifically retinal fluid, in the management of nAMD in Canadian daily practice. The objective is to provide a set of easy-to-follow guidelines (Table 1) and algorithms that will streamline the management of nAMD and optimize visual outcomes.

\section{Methodology}

The development of these expert recommendations began with a systematic literature review. Retina specialists were assigned a specific imaging- or treatment-related topic. These topics were further discussed during a meeting in Toronto, ON, Canada, on September 21, 2019. The retina specialists also reflected upon the Canadian consensus paper about the management of nAMD published in 2012 [12].

\section{Identification and Classification of Neovascularization in AMD: Which Imaging Modality and When?}

With advancements in imaging based on fluorescein angiography (FA) and OCT, a new classification arose that categorized lesions as type 1 (subretinal pigment ep- ithelium [RPE]), type 2 (subretinal), type 3 (intraretinal), or mixed neovascularization [13]. Polypoidal choroidal vasculopathy $(\mathrm{PCV})$ was considered to be a special form of type 1 nAMD, while occult, classic, and retinal angiomatous proliferation (RAP) corresponded to types 1,2 , and 3 , respectively $[13,14]$. According to an OCT-based analysis, $53 \%$ of patients were diagnosed with type 1 choroidal neovascularization (CNV) and $47 \%$ with type 2 CNV; type 3 (RAP) was not assessed [15].

\section{Fluorescein Angiography}

Traditionally, FA has been considered the gold standard for the diagnosis of CNV. FA patterns observed in AMD patients can be classified as hypo- or hyperfluorescent (Table 2) [16]. Although classification of CNV lesions as classic and occult became obsolete with newer imaging modalities, leakage on FA still is an important diagnostic feature indicating an active disease process.

\section{Indocyanine Green Angiography}

Indocyanine green angiography (ICGA) provides high-quality images of the choroidal circulation and can reveal occult CNV that may be undetectable on FA [17]. With a longer operating wavelength (795-805 nm peak fluorescence emission), ICGA has the advantage of superior fluorescence through pigment, fluid, lipid, and hemorrhage than fluorescein dye, allowing deeper imaging into the retina [18]. Although ICGA is not widely used or available in Canada due to limited access to the dye and/ or high costs [19], this imaging modality may be especially helpful to detect PCV, reevaluate nonresponding patients, and for conditions such as central serous chorioretinopathy which may require a different therapeutic approach from that used in $\mathrm{AMD}$.

CNV appears on ICGA as a plaque, a focal hot spot, or a combination of both. Plaques (61\% of cases) $[20,21]$ are usually formed by late-staining vessels, often correspond to occult $\mathrm{CNV}$, and have a poor visual prognosis. The focal "hot spots" (approx. 30\% of cases; Fig. 1) have a better prognosis and are potentially treatable by ICGA-guided laser photocoagulation [22].

FA and ICGA are invasive techniques that require intravenous dye injection, which can result in hepatic, renal, and allergic complications [23]. The incidence of adverse reactions is $0.05 \%$ for ICG and $5 \%$ for fluorescein [24]; however, FA is routinely performed in patients with advanced diabetic retinopathy, many of whom have diabetes-related renal compromise. Cases of hypotensive shock and death have been reported [25]. 
Fluorescein angiography

Comprehensive evaluation of a patient with suspected nAMD should include FA. However, due to considerations regarding reimbursement and other social factors, a combination of clinical examination, SD-OCT, and fundus photography is usually sufficient to diagnose nAMD with associated leakage.

As a part of multimodal imaging modalities, FA has significant relevance in the management of nAMD, especially at time of diagnosis. Baseline FA may also be a useful benchmark reference for future treatment assessments (i.e., whether and how a patient is responding to therapy).

Reassessment of FA may be helpful in the following situations:

- when an increase in macular fluid is seen on OCT during anti-VEGF treatment in a patient whose disease has been stable/ controlled

- in the presence of new hemorrhage

- in situations when vision decreases without other clinical signs

- in the presence of intraocular inflammation, retinal vasculitis, or retinal vascular occlusion

The new technologies, including OCT-A, are faster and less invasive but should continue to be used in conjunction with FA. In the era of SD-OCT, the utility of FA has diminished.

- The usefulness and necessity of FA was underscored by the limited sensitivity and specificity for fluid associated with TD-OCT; TD-OCT should therefore always be used in combination with FA

Indocyanine green angiography

PCV is a clinical diagnosis that is optimally confirmed with ICGA. ICGA is crucial to most accurately define the location of all polyps (OCT-A is also proving valuable in this context).

ICGA may also be helpful in patients with PCV who are to be treated with anti-VEGF in combination with PDT. In Canadian clinical practice, however, clinicians often forego ICGA for practical reasons (limited access to dye or equipment), and use clinical assessment and other imaging modalities to guide PDT.

Spectral-domain optical coherence tomography

In current clinical practice, SD-OCT is the new standard of care for the diagnosis and management of nAMD and the main driver for treatment decisions. It is particularly useful for determining and monitoring the fluid compartments of the retina.

OCT should be performed initially and then every time a decision is to be made about whether a treatment is needed or not (pro re nata), or regarding the time interval between treatments (T\&E). Once a treatment interval has been established, OCT should be used to monitor for recurrent disease activity at predetermined intervals.

Optical coherence tomography angiography

OCT-A is an emerging imaging modality, that, with time and improvements in image acquisition, may become the standard of care. - Currently, it is not essential for the effective management of patients with nAMD; however, it can be a useful tool for physicians who make retreatment decisions in complex cases (i.e., patients not responding to anti-VEGF, recurrence of fluid in stable/ controlled patients, etc.) without the use of an angiogram. OCT-A may become very useful in classifying nAMD into type 1, 2, or 3 (RAP), as clinical experience with this modality evolves.

- For those who already use angiography to inform their treatment decisions, OCT-A can be a useful complementary technique that provides additional information.

Fundus autofluorescence

In isolation, FAF is not useful for screening, diagnosing, or monitoring $\mathrm{nAMD}$, but it complements other imaging modalities.

- FAF may be useful in detecting subclinical disease, which is of relevance when monitoring the fellow eye for signs of AMD.

- It is also of particular value for monitoring the presence and progression of GA and identifying RPE tears.

- Sensitivity and specificity of FAF, compared to other modalities, have yet to be determined.

Multimodal imaging

Comprehensive evaluation of patients with nAMD should include multimodal imaging. One modality is often insufficient for thorough investigation, which should include staging of the disease to ascertain prognosis and subsequent decision-making. However, with ongoing improvements in technologies and the quality of obtained images, in the majority of cases, adequate information can be obtained using SD-OCT with clinical examination and/or color fundus photography.

- SD-OCT is an indispensable tool to evaluate, treat, and monitor nAMD.

- After the loading phase, if there is no change from baseline in fluid after a minimum of 2 consecutive follow-up visits in patients treated with anti-VEGF, other imaging modalities should be considered to assess the underlying cause.

Fluid and its impact on therapeutic decisions

An increase in fluid is a sign of disease activity and should be treated regardless of its location.

- Foveal IRF is a negative predictor of VA outcomes and needs to be treated early and aggressively

- Patients with foveal SRF usually respond well to anti-VEGF therapy. These patients may be good candidates for the T\&E approach Sustained drying of the retina (defined as $\geq 2$ consecutive fluid-free visits) is an indicator of better disease control and may be associated with improved long-term outcomes.

Fluctuations in CST during anti-VEGF therapy are associated with worse visual outcomes and have a negative prognostic value; patients may require further reevaluation and consultations.

CST, central subfield thickness; FA, fluorescein angiography; FAF, fundus autofluorescence; GA, geographic atrophy; ICGA, indocyanine green angiography; IRF, intraretinal fluid; nAMD, neovascular age-related macular degeneration; OCT, optical coherence tomography; OCT-A, OCT angiography; PCV, polypoidal choroidal vasculopathy; PDT, photodynamic therapy; RAP, retinal angiomatous proliferation; RPE, retinal pigment epithelium; SD, spectral domain; TD, time domain; T\&E, treat-and-extend; VEGF, vascular endothelial growth factor. 
Table 2. Causes of hypofluorescence and hyperfluorescence

\begin{tabular}{ll}
\hline Causes of hypofluorescence & Causes of hyperfluorescence \\
\hline Blocked & Transmitted fluorescence \\
Intraretinal or subretinal hemorrhage/exudate & RPE atrophy \\
Sub-RPE hemorrhage & RPE rip \\
Pigment proliferation & Hard, basal laminar drusen \\
Pigment epithelial clumping (RPE rip) & \\
& Extravascular fluorescence \\
Vascular filling defect & Serous pigment epithelial detachment \\
Choroidal vascular atrophy & Soft drusen \\
Retinal capillary occlusion & Disciform scar \\
& Loculated fluid \\
& Cystoid macular edema \\
& Abnormal vessels \\
& Choroidal neovascularization \\
& Retinal angiomatous proliferation \\
\hline
\end{tabular}

Data are from [16] with the authors' permission. RPE, retinal pigment epithelium.

\section{Spectral-Domain OCT}

Over the past decade, spectral-domain (SD)-OCT has been adopted as the gold standard for the diagnosis, follow-up, and management of nAMD CNV. It is a noninvasive, highly reproducible, high-resolution imaging tool that allows for easy visualization of anatomic changes that can be associated with angiographic leakage [26, 27].

Improvements in acquisition speed and image analysis make SD-OCT vital in decision-making for the diagnosis and treatment of nAMD. With active nAMD, SD-OCT can be used to detect signs of activity and to establish a baseline retinal thickness, volume, and fluid involvement. Additionally, SD-OCT is helpful in identifying the location and level of CNV (intraretinal, subretinal, and subRPE) and other lesion components (blood, fluid, pigment, and fibrosis) $[28,29]$. Detection of shallow, irregular RPE elevation ([SIRE] comprising RPE increases with a greatest transverse linear dimension $\geq 1,000 \mu \mathrm{m}$, an irregular RPE layer, a height that is primarily $<100 \mu \mathrm{m}$, and a nonhomogeneous internal reflectivity) was found to be characteristic of the "double layer sign" on SD-OCT in the presence of nonexudative macular neovascularization (NE-MNV) [30]. The authors recommended that SIRE be used as a screening tool for NE-MNV on routine OCT. $\mathrm{SD}-\mathrm{OCT}$ is also valuable for the early detection of type 3 MNV before it reaches the exudative stage [31].

Three-dimensional SD-OCT volume scans are interpolated from parallel 2-dimensional OCT scans (B-scans) [32]. Current OCT devices allow for adjustment of the density of these B-scans by changing the interscan distance (ISD). Higher B-scan density results in a higher transverse resolution of the generated volume scan but also in a prolonged acquisition time, time for electronic data transfer, and the time for data reviewing. A recent study demonstrated that ISD of 60,120 , or $240 \mu \mathrm{m}$ did not make a significant difference in the detection of treatment-relevant exudative retinal changes during monitoring of the response to intravitreal therapy of macular diseases [33]. SD-OCT can also be useful for discerning foveal morphologic changes that precede CNV formation [34]. These include new RPE or new photoreceptor defects, drusen touching the photoreceptor layer and the external limiting membrane, new drusen, and hyperreflective spots that probably represent new growing vessels.

En face swept-source (SS)-OCT is a promising rapid, noninvasive, high-resolution technology [35]. There is a correlation between angiography and en face SS-OCT images in nAMD. However, SS-OCT is not currently considered a standard requirement for nAMD monitoring.

\section{OCT Angiography}

OCT angiography (OCT-A) is a noninvasive technique for imaging the microvasculature of the retina and the choroid, potentially eliminating the need for intravascular dyes [36]. The same area is repeatedly scanned and differences are analyzed between scans over time. This allows the detection of zones containing high flow rates (i.e., changes between scans) and zones with slower or no flow at all [37]. While OCT-A offers great potential, there remains a need for a better understanding of the range of 


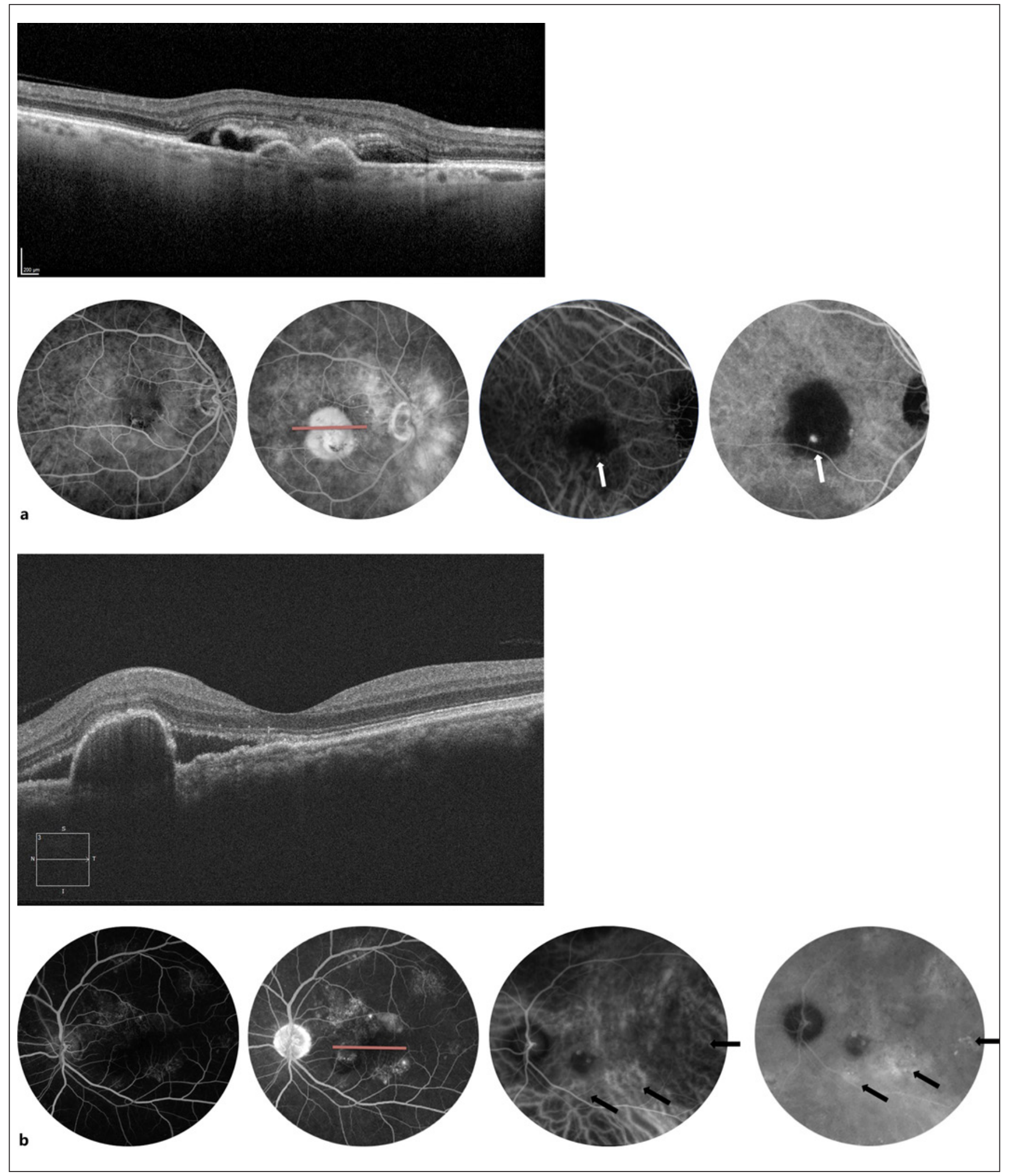

Fig. 1. FA versus ICGA. a Retinal angiomatous proliferation. b Polypoidal choroidal vasculopathy. White arrows indicate a hot spot; black arrows indicate polyps. Images courtesy of A.S. and D.T.W. FA, fluorescein angiography; ICGA, indocyanine green angiography. 


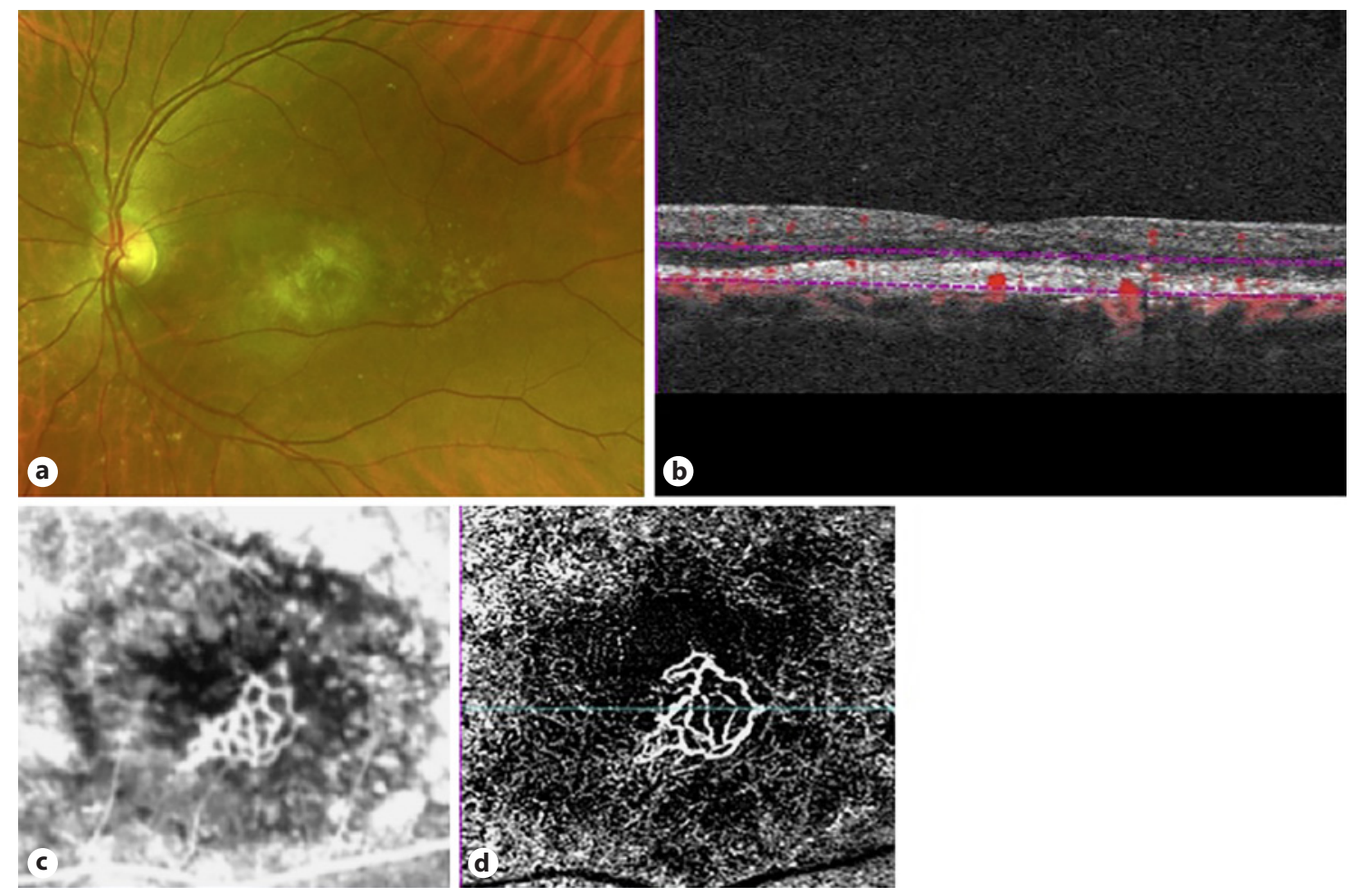

Fig. 2. Example of multimodal CNV analysis. a Retinal photograph. b OCT-B scan with flow in red. The horizontal green line seen in panel D marks the section of the OCT-A B-scan image. c FA. d En face OCT-A. Images courtesy of D.T.W. CNV, choroidal neovascularization; FA, fluorescein angiography; OCT-A, OCT angiography.

technologies and methods to interpret the images [38]. As clinical trials have been designed without OCT-A-based end points, caution is required when making treatment decisions based on OCT-A imaging alone.

OCT-A is not without limitations, including artifacts that may affect the images $[39,40]$. For example, the automatic segmentation of vascular layers may be challenging in eyes with myopia, even in the absence of chorioretinal complications. Projection artifacts may affect the visualization of the deeper vascular layers. Shadowing artifacts occur when the OCT beam is weakened or blocked, thereby hindering its passage to the deeper layers of the retina. OCT-A is also limited in the visualization of the larger choroidal vessels, typically displayed as silhouettes, with complete loss of signal at greater depths [41]. OCT-A also does not show leakage that can be seen in intravenous FA studies.

Up to $95 \%$ agreement has been demonstrated between different CNV patterns identified on OCT-A and treatment decisions based on conventional multimodal imaging [42]. OCT-A is particularly useful for detecting branching vessels, vascular loops, peripheral anastomosis, hypointense choriocapillaris, and subclinical CNV
(Fig. 2) [43]. However, it is not as useful for detecting PCV and RAP lesions [44]. In addition, interpretation of OCT-A scans takes time, which increases the burden on the already-busy Canadian retina practice.

\section{Fundus Autofluorescence (FAF)}

FAF is a rapid, noninvasive retinal imaging modality that provides a density map of lipofuscin, the predominant ocular fluorophore, in the RPE [45]. FAF is especially helpful for monitoring the progression of atrophy in nAMD cases. In early non-nAMD, FAF may show widespread hyper- and hypoautofluorescent areas. Drusen have variable appearance on FAF depending on their size and composition and the health of the overlying RPE. High-risk drusenoid pigment epithelial detachments (PEDs) appear as foci of hyperautofluorescence bordered by a hypoautofluorescent halo [46]. Reticular pseudodrusen appear as elongated foci of hypoautofluorescence bound by interspersed hyperautofluorescence [47]. Reticular pseudodrusen are associated with a high risk of progression to advanced disease, including $\mathrm{CNV}$ and/or geographic atrophy [48]. 

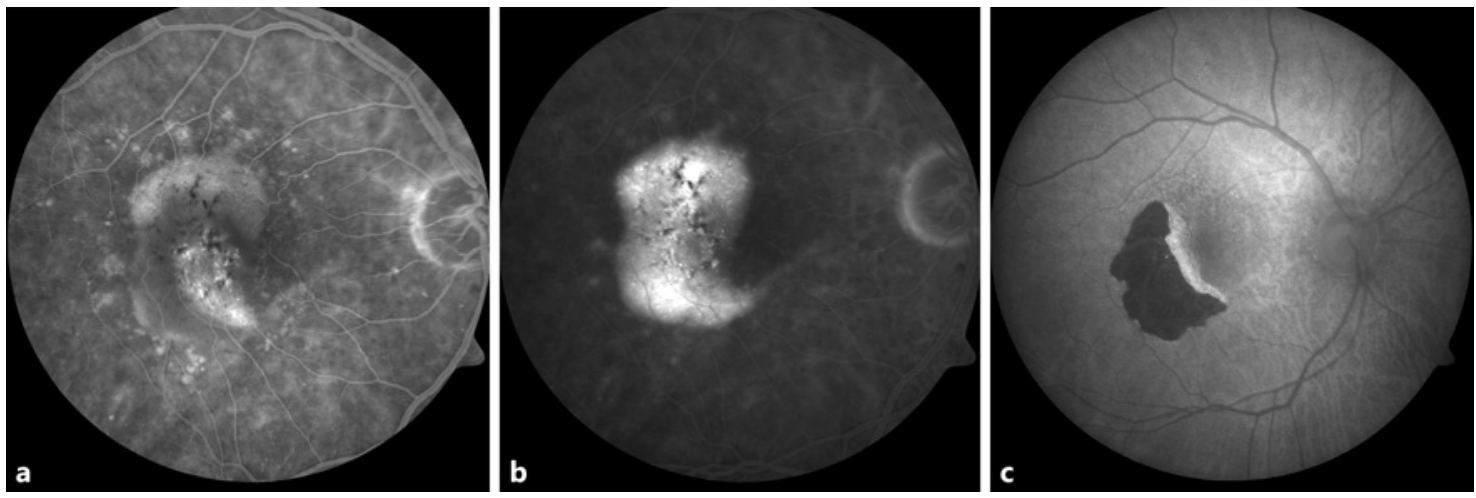

Fig. 3. FA showing an RPE detachment with subsequent RPE rip shown on FAF. a Early FA showing PED. b Late FA showing PED. c FAF performed 1 month later showing RPE tear. Images courtesy of S.B. FA, fluorescein angiography; FAF, fundus autofluorescence; PED, pigment epithelial detachment; RPE, retinal pigment epithelium.

Table 3. Imaging modalities for nAMD

\begin{tabular}{|c|c|}
\hline Imaging modality & Recommendations ${ }^{\mathrm{a}}$ regarding utility \\
\hline ICGA & $\begin{array}{l}\text { - May be helpful in patients with PCV when they are to be treated with PDT } \\
\text { - Reduced use in Canada due to the low availability of ICG dye }\end{array}$ \\
\hline OCT-A & $\begin{array}{l}\text { - Allows for identification of subclinical features (membranes) not seen by other modalities } \\
\text { - Useful for detecting serous PED, branching vessels, vascular loops, peripheral anastomosis, hypointense } \\
\text { choriocapillaris } \\
\text { - Not as useful for detecting PCV or leakage } \\
\text { - Unnecessary for standard of care }\end{array}$ \\
\hline
\end{tabular}

$\mathrm{AF}$, autofluorescence; $\mathrm{AMD}$, age-related macular degeneration; $\mathrm{CNV}$, choroidal neovascularization; FA, fluorescein angiography; FAF, fundus autofluorescence; ICGA, indocyanine green angiography; OCT, optical coherence tomography; OCT-A, OCT angiography; PCV, polypoidal choroidal vasculopathy; PDT, photodynamic therapy; PED, pigment epithelial detachment; RPE, retinal pigment epithelium; SD, spectral domain; TD, time domain; VEGF, vascular endothelial growth factor.

${ }^{a}$ Recommendations are based on the Canadian landscape.

$\mathrm{b}$ Continuous presence of fluid despite treatment, new hemorrhage, unexplained decrease in vision, increase in disease activity in patients previously well-controlled, RPE tear, etc.

${ }^{c}$ Clinical significance has yet to be confirmed (i.e., frequency of treatment, benefits of earlier intervention, etc.).

d Intensity may change with circadian rhythms, adaptation to the dark, and photoreceptor "bleaching." 


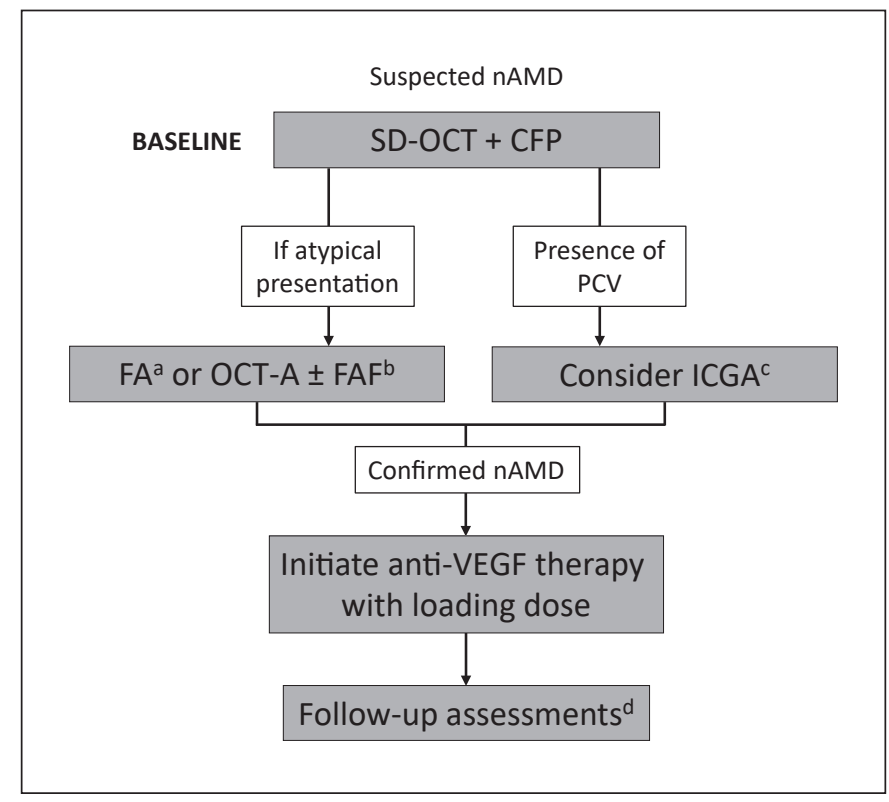

Fig. 4. Diagnostic algorithm for nAMD, based on available imaging modalities. ${ }^{\mathrm{a}}$ In the era of SD-OCT, the necessity and usefulness of FA has diminished. ${ }^{b}$ To assess for geographic atrophy. ${ }^{c}$ If considering photodynamic therapy. ${ }^{\mathrm{d}}$ For the follow-up assessments, it is recommended that one continues with the imaging modality that was used for the initial diagnosis to appropriately assess and record changes. In the case of atypical presentation(s) or unusual findings, other ancillary modalities can be used to determine the course of the disease. With ongoing improvements in technologies and the quality of the images, in the majority of cases, adequate information can be obtained in the office using SD-OCT and CFP. CFP, color fundus photography; FA, fluorescein angiography; FAF, fundus autofluorescence; ICGA, indocyanine green angiography; OCT-A, OCT angiography; PCV, polypoidal choroidal vasculopathy; SD, spectral domain.

In the early stage of nAMD, FAF usually appears normal because the activity of RPE cells remains preserved [45]. Classic and occult type 1 neovascularization usually appears hypoautofluorescent due to the blockage or atrophy of the overlying RPE. However, CNV may be bordered by hyperautofluorescence due to RPE proliferation or photoreceptor loss [49]. Hemorrhages and exudates are initially hypoautofluorescent due to excitation light absorption, but may become hyperautofluorescent after undergoing organization [45]. PED can present as a cartwheel-like configuration with increased and decreased levels of signal intensity (Fig. 3). RPE tears are readily shown with FAF.

Limitations of FAF include low signal strength and autofluorescence artifacts from the anterior segment. Software to analyze and quantify hypo- or hyperautofluores- cence intensity has been developed but is not readily available. FAF intensity may change with circadian rhythms (autofluorescence decreases at the end of the day), adaptation to the dark, and photoreceptor "bleaching."

A multimodal imaging approach is required for the diagnosis of different subtypes of neovascularization. Recommendations for the use of various imaging modalities in the management of nAMD in Canada are provided in Table 3 and a diagnostic algorithm is shown in Figure 4. For follow-up assessment and monitoring, it is recommended one continues with the imaging modality that was used for the initial diagnosis, so as to appropriately assess and record changes. In the case of atypical presentation(s) or unusual findings, ancillary modalities can be used to determine the course of the disease.

\section{Imaging Biomarkers and Their Prognostic Value in nAMD}

Over the past decade, several imaging biomarkers appeared to have predictive and prognostic value in the management of nAMD. Further research into a definitive association between prognostic markers such as subretinal hyperreflective material, atrophy, fibrosis, and predictive outcomes is required.

\section{Retinal Thickness}

The measurement of retinal thickness is the easiest method to quantify retinal changes on OCT [48]. Continued increase in retinal thickening indicates leakage in the area. Integrated automated software algorithms are capable of segmenting the internal limiting membrane and the RPE or Bruch's membrane as the inner and outer retinal boundaries. The average thickness in a circular field ( $1 \mathrm{~mm}$ in diameter) centered on the fovea is used as a quantitative outcome measure in clinical trials and to assess treatment response in clinical practice. The main limitation of retinal thickness as a biomarker is that it includes an array of retinal compartments, and the simple measurement of central retinal thickness does not differentiate the contribution of these components to pathological changes and visual outcomes [50].

\section{Intraretinal Fluid}

On OCT, intraretinal fluid (IRF) appears as diffuse retinal thickening (i.e., $\geq 100 \mu \mathrm{m}$ increase in retinal thickening indicates fluid) or as hyporeflective cystoid spaces, depending on the imaging quality. IRF usually indicates 
that the neovascular network has breached from its confined sub-RPE space and has started to infiltrate the neurosensory retina. Thus, IRF is a sign of a more aggressive lesion type or a sign of late presentation in chronic occult $\mathrm{CNV}$. In treatment-naïve nAMD patients, the rate of IRF findings at the initial presentation ranges from 52 to $76 \%$ [51-53]. IRF at presentation may predict a delayed response to treatment and has been associated with a poorer prognosis [50].

One should differentiate between cystoid fluid secondary to exudative CNV ("exudative intraretinal cyst" [IRC]) and that associated with neurosensory degeneration ("degenerative IRC"). While exudative IRCs are relatively large ovoid spaces overlying PED (type 1 or 3 lesions) or neovascular tissue (type $2 \mathrm{CNV}$ ), degenerative IRCs are generally small, square, sharply demarcated, hyporeflective spaces over dysfunctional RPE [50,54]. Lai et al. [54] differentiated degenerative from exudative IRCs if they had 2 of the following 3 morphological criteria: alteration of the underlying RPE layer, a square-shaped lesion with $>1$ concave or straight border, and small size of the IRC (greatest dimension $<125 \mu \mathrm{m}$ ) without obvious expansion of adjacent layered retinal structure. Degenerative IRCs are not a sign of active disease and do not respond to anti-VEGF treatment, with resultant poor visual improvement up to 12 months after treatment initiation [54-56].

\section{Subretinal Fluid}

Subretinal fluid (SRF) is associated with all lesion types and is typically the first exudative sign in type 1 lesions [51]. It is the only biomarker that is consistently associated with a positive response to anti-VEGF treatment. In addition, eyes with baseline SRF are less likely to develop macular atrophy even when undergoing an intensive monthly anti-VEGF regimen $[57,58]$. Although patients with baseline SRF derive larger VA benefits from antiangiogenic treatment than those without SRF, one should keep in mind that recurrence of SRF is a sign of disease activity and, if left untreated, may have a negative impact on visual outcomes $[59,60]$. On the other hand, in the FLUID study, tolerance of some SRF $(<200 \mu \mathrm{m}$; relaxed arm) did not adversely affect visual outcomes; however, the presence of IRF in a higher proportion of patients in the relaxed treatment group at month 24 suggests that SRF may lead to IRF over time [61].

\section{Sub-RPE Fluid}

Studies suggest that changes in the sub-RPE spaces potentially reflect the primary disease activity in serous and vascularized PED $[57,62]$, with the sub-RPE space a predictor of recurrence after the loading phase with antiVEGF [63]. This indicates that the changes in the subRPE space could be a sensitive marker to monitor disease activity. A recent clinical trial identified sub-RPE fluid in $43 \%$ of subjects [5]. Spatial correlation for PED was found to be higher for cystoid IRF than for SRF [64].

\section{Pigment Epithelial Detachment}

Traditionally, clinicians have not included PED in the decision algorithms for treatment indications, and none of the large-scale prospective treatment trials have included it in their retreatment criteria. Recent studies revealed that $\mathrm{PED}$-associated neovascular reactivations may be implicated in long-term vision loss in patients treated with individualized anti-VEGF regimens [50]. A larger PED is also an important risk factor for the development of RPE tears, a severe complication in nAMD with often poor functional outcomes [65]. A domeshaped PED is associated with lower central macular thickness at presentation, but it does not appear to affect the response to treatment [66].

\section{Central Subfield Thickness}

Fluctuations in central subfield thickness (CST) during anti-VEGF therapy are associated with worse visual outcomes and a negative prognostic value [67]. Pooled analysis of the Comparison of the AMD Treatment Trials (CATT) and Inhibit VEGF in Age-Related Choroidal Neovascularisation (IVAN) trial demonstrated that CST fluctuation after the loading phase is associated with poor visual outcomes [68]. Although the amplitude of VA gain is individual, data from these 2 analyses indicate that a more stable CST reflects better disease control and the associated better visual outcomes $[67,68]$.

\section{Fluid and Its Impact on Therapeutic Decisions}

A 5-year follow-up of the CATT demonstrated that $60 \%$ of the eyes studied had IRF, $38 \%$ had SRF, and $36 \%$ had sub-RPE fluid [69], potentially due to undertreatment. Considering the evidence demonstrating the impact of fluid on long-term visual outcome, the management of fluid presents a significant unmet medical need. Any increase in fluid seen on OCT may indicate disease activity and should be further evaluated, and, if confirmed to be associated with neovascularization, treated regardless of its location. 


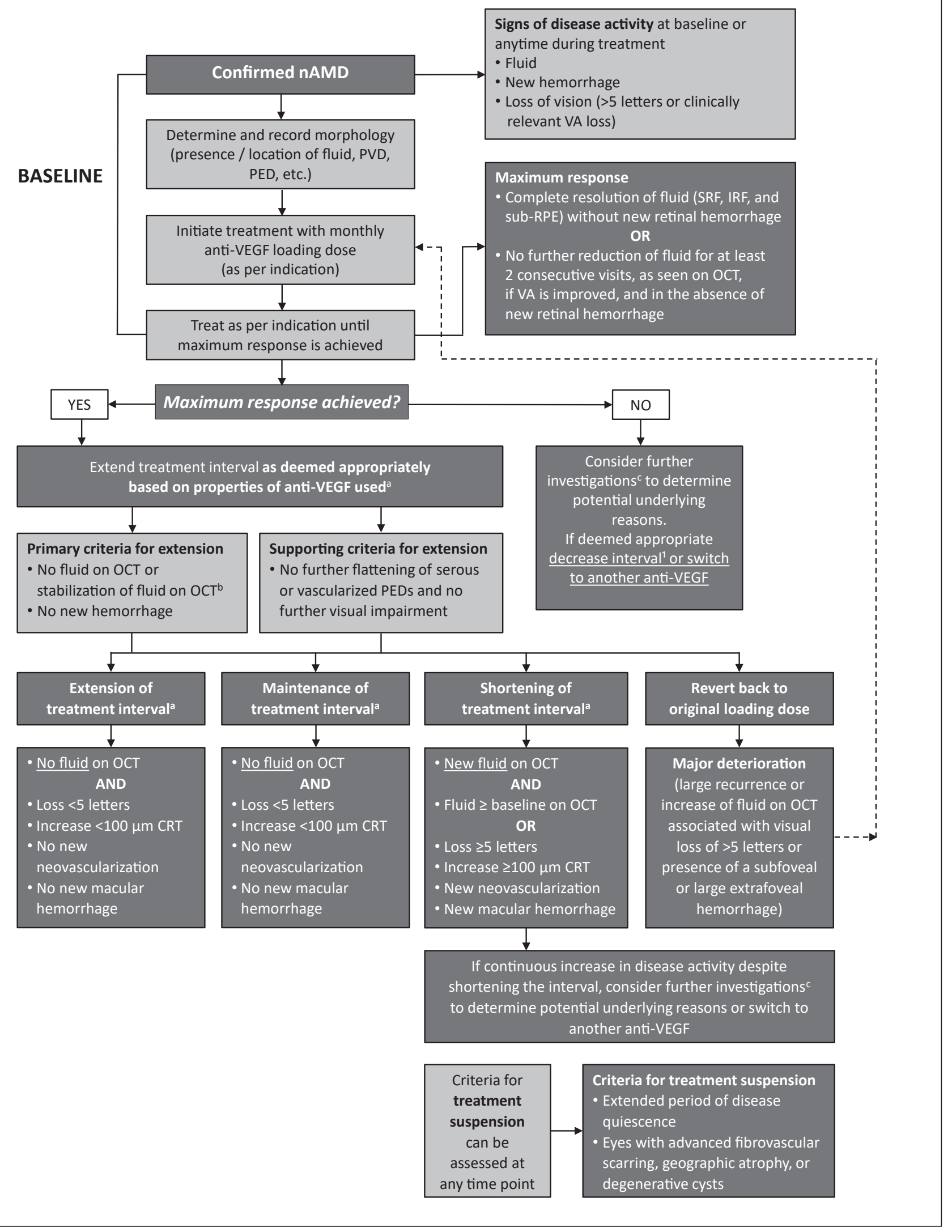

(For legend see next page.) 
As foveal SRF responds well to anti-VEGF treatment, patients with SRF should be monitored closely and treated accordingly. SRF in nAMD seems to positively influence not only visual function but also the need for retreatment. In a post hoc analysis comparing the outcomes of fixed frequent and infrequent anti-VEGF treatments, eyes with SRF had similarly favorable VA outcomes regardless of the treatment regimen $[61,70]$. Current data suggest that eyes with SRF at presentation are likely to exhibit a disease course that can be managed with anti-VEGF injections with extended intervals up to every 12 weeks. Eyes with SRF may be the ideal candidates for T\&E regimens; however, these patients still require close monitoring and appropriate management of retinal fluid. Evidence that SRF indicates a good prognostic VA outcome should be interpreted with caution, as any fluid seen on OCT may indicate disease activity, and the end goal should always be a dry retina. Current guidelines recommend retreatment with antiVEGF in the presence of disease activity, defined as IRF, SRF, or sub-RPE fluid or hemorrhage, lesion growth, or visual deterioration [71-73]. Furthermore, the volume of SRF appears to be an independent predictor of response [74]. Patients with greater baseline SRF thickness require more injections than patients with SRF $<118 \mu \mathrm{m}$. Sustained retinal drying (defined as $\geq 2$ consecutive fluid-free visits) is an indicator of better disease control and may be associated with improved long-term outcomes. Figure 5 depicts a fluid-based treatment algorithm for nAMD. Although FA is the gold standard for diagnosis of $\mathrm{CNV}$ in clinical trials, access to this modality in real-world clinical practice is becoming increasingly difficult; OCT-based criteria have therefore been adopted.

Fig. 5. Proposed fluid-based treatment algorithm for nAMD. ${ }^{a}$ Globally, the most common algorithm used is treat-and-extend (T\&E). When deciding upon treatment interval, maximal duration of the initial monthly treatment period, and maximal extension, consider the underlying morphology (determined at baseline) and the pharmacodynamics/pharmacokinetics of the anti-VEGF used. For example, shorter interval for eyes with features associated with negative prognosis (see descriptions in the text). Maximal extension should be driven by properties of anti-VEGF. ${ }^{\mathrm{b}}$ For at least 2 consecutive injections. ${ }^{c}$ Consider different imaging modalities and/or referral for second opinion/peer-to-peer consultation. CRT, central retinal thickness; IRF, intraretinal fluid; PED, pigment epithelial detachment; PVD, posterior vitreous detachment; RPE, retinal pigment epithelium; SRF, subretinal fluid; VA, visual acuity.

Imaging Biomarkers in nAMD

\section{Management of PCV and RAP}

Left untreated, symptomatic PCV may lead to severe vision loss, so active intervention is strongly indicated [19]. Current treatment options for PCV include antiVEGF and verteporfin photodynamic therapy (PDT). While patients with branching vascular networks usually respond to anti-VEGF therapy, those with solitary polyps tend to be anti-VEGF-resistant and responsive to PDT, used alone or as an adjunct to anti-VEGF therapy [75]. Anti-VEGF agents improve visual function by restoring normal retinal thickness and reducing the reuptake of VEGF after PDT, while PDT facilitates polyp regression. The efficacy of these treatments as monotherapy versus combination therapy was evaluated in the EVEREST trial [76], which demonstrated that verteporfin PDT, either alone or combined with ranibizumab $0.5 \mathrm{mg}$, was superior to ranibizumab monotherapy in achieving complete regression of polyps in patients with symptomatic macular PCV. In the Aflibercept in Polypoidal Choroidal Vasculopathy (PLANET) trial [77], aflibercept monotherapy was associated with improved visual and/or functional outcome in $>85 \%$ of the PCV patients. The benefit of adding PDT could not be determined due to the low number of patients who met the criteria to receive it.

Patients with RAP usually respond well to anti-VEGF therapy with or without PDT [78-80]. Other approaches include laser photocoagulation of feeder vessels, or intravitreal triamcinolone to reduce exudation (intraretinal/ subretinal), followed 7-14 days later by PDT [81].

\section{Therapeutic Options and Goals in 2021 and Beyond}

The treatment goal for nAMD is to achieve the best possible VA benefits through early initiation of therapy, which is then maintained through continuous timely disease monitoring and retreatment. A proactive treatment approach, where assessment and retreatment occur during the same visit, minimizes the need for additional visits and alleviates the burden on the treating clinicians and patients.

When applied according to the approved dosing, treatment extension allows for injection intervals to be increased gradually in a stepwise fashion to maintain stable visual and anatomic outcomes [7, 8, 82]. Extension may be considered for eyes without macular fluid on OCT and stable vision. The main criteria for shortening treatment intervals are persistent macular fluid with stable vision, recurrent fluid, decrease in vision in the presence of fluid,

Ophthalmologica 2021;244:265-280 
new macular hemorrhage, new $\mathrm{CNV}$, or any other sign(s) of exudative disease activity considered vision-threatening in the opinion of the treating clinician [8].

Currently, 3 anti-VEGF therapies are available globally, 2 of which (ranibizumab and aflibercept) are approved for intravitreal use. Bevacizumab, an anti-VEGF agent indicated for the treatment of some forms of cancer, is reconstituted and used off-label to treat nAMD. The use of these agents in clinical practice has further improved the understanding about the disease, its natural history, and the need for individualized treatment. Aflibercept's every-8-week (q8w) dosing schedule for the treatment of wet AMD (following 3 monthly loading doses) offered a promise of longer interval between treatments; however, some patients were found to require more frequent doses to maintain their VA gains. The "sawtooth" pattern in retinal thickness observed in the VIEW trials after the loading doses hinted at the necessity for a shorter dosing schedule [83]. Thus, the search continues for a therapeutic approach that will lead to a sustainability of VA gains and a reduced number of visits/ treatments.

Brolucizumab has recently been approved for the treatment of patients with nAMD in some countries. This single-chain anti-VEGF antibody fragment binds to all isoforms of VEGF-A and blocks their action [84]. The smaller molecular size of brolucizumab $(28 \mathrm{kDa}$, vs. 48 $\mathrm{kDa}$ for ranibizumab and $115 \mathrm{kDa}$ for aflibercept) allows for the delivery of a higher molar dose than is possible with larger molecules, thereby offering the potential of more effective tissue penetration [85]. This superior penetration is believed to confer better fluid control across all layers of the retina, resulting in similar visual outcomes and better anatomical outcomes. The phase $3 \mathrm{HAWK}$ and HARRIER trials compared brolucizumab ( 3 and $6 \mathrm{mg}$ given as 3 monthly loading doses at 0,4 , and 8 weeks, and then every 8 or 12 weeks depending on disease activity) to aflibercept (2 mg q8w) [5]. At 48 weeks, noninferiority of BCVA compared to aflibercept (the primary outcome) was achieved with both 3- and 6-mg doses. Brolucizumab was also significantly superior in achieving a dry macula, with more reduction in IRF, SRF, and sub-RPE fluid at weeks 16, 36, and 48. Early postmarketing reports of vasculitis, including retinal occlusive vasculitis, were confirmed by an external safety review committee as a rare (15.47/10,000 injections) safety signal [86, 87].

Promising approaches in advanced phases of clinical development include a bispecific monoclonal antibody (faricimab) and a port delivery system (PDS) with ranibizumab.
Faricimab is the first bispecific antibody designed for intravitreal use to simultaneously neutralize both angiopoietin-2 (Ang-2) and VEGF-A with high potency and specificity [88]. In nAMD, Ang-2 works synergistically with VEGF to drive a decrease in the abnormal blood vessel growth and fluid leakage which contribute to vision loss. Ang-2 also plays an important role in multiple aspects of inflammation in nAMD $[89,90]$. Topline results from the phase 3 TENAYA and LUCERNE studies showed that faricimab at fixed intervals of up to every 16 weeks achieved noninferior VA outcomes to those for aflibercept every 8 weeks [91].

The PDS with ranibizumab, a small, refillable eye implant (approx. $3 \mathrm{~mm}$ in length) was designed to allow patients with nAMD to avoid retreatment for several months. The device is placed through a $3.5-\mathrm{mm}$ scleral incision in the pars plana. Topline results from the phase 3 Archway study found that $98.4 \%$ of PDS patients were able to achieve $\geq 6$ months without additional treatment and had visual outcomes comparable to those who received monthly intravitreal ranibizumab [92]. An openlabel extension to the PORTAL study was initiated in September 2018 and is ongoing [93].

\section{Conclusions}

nAMD is a chronic, life-long condition requiring ongoing treatment with regular follow-up and monitoring to control exudative disease activity. The frequency of follow-ups can pose a significant burden for patients, clinicians, and the healthcare system. To reduce this burden, many clinicians currently rely on imaging biomarkers, particularly the presence of retinal fluid, to adjust treatment frequency according to disease activity. Although recent evidence indicates that the location of fluid carries a prognostic value with regard to the response to treatment and visual outcomes, it is important to keep in mind that the presence of any fluid may indicate disease activity and needs to be appropriately treated. Sustained drying of the retina is an indicator of better disease control and may be associated with improved long-term outcomes. Thus, clinicians should opt for treatments with the ability to improve dry retina and are then less likely to require shortening of the treatment interval. The ultimate treatment goal for nAMD in 2021 and beyond should be the maintenance of good VA with less frequent visits/injections for all patients. 


\section{Acknowledgement}

The authors wish to acknowledge Radmila Day and Jeff Alexander from SNELL Medical Communication for their medical writing assistance.

\section{Conflict of Interest Statement}

The authors report the following details regarding affiliation or involvement in an organization or entity with a financial or nonfinancial interest in the subject matter or materials discussed in this article. D.T.W. is an investigator in active clinical trials for Novartis Pharmaceuticals Canada and Roche Canada, has received research funding from Bayer Canada, Novartis Pharmaceuticals Canada, and Roche Canada, and been a consultant for Alcon, Allergan, Bayer Canada, Bausch Health, Novartis Pharmaceuticals Canada, Roche Canada, Topcon Canada, and Zeiss; he also owns equity in ArcticDx. A.R.B. has been a consultant and received honoraria from Bayer Canada, Novartis Pharmaceuticals Canada, Roche Canada, and Allergan. S.B. has been a consultant for Allergan, Bayer Canada, and Novartis Pharmaceuticals Canada, and participated in clinical studies founded by Bayer Canada and Novartis Pharmaceuticals Canada. J.C. has been a consultant and advisory board member for Allergan, Bayer Canada, and Novartis Pharmaceuticals Canada, and received research support from Novartis Pharmaceuticals Canada and Bayer Canada. K.C. has been a consultant/advisory board member for Bayer Canada and Novartis Pharmaceuticals Canada, and received research support from Novartis Pharmaceuticals Canada. A.F.C. has received honoraria for his performance of consultant and speaking roles for both Novartis Pharmaceuticals Canada and Bayer Canada within the past year. R.I.D. has been a consultant/advisory board member, and received speaking honoraria from Novartis Pharmaceuticals Canada, Alcon, Bayer Canada, Allergan, and Bausch and Lomb. D.G. is a consultant and speaker for Novartis Pharmaceuticals Canada, Bayer Canada, and Allergan. B.H. has received speaker honorarium from Alcon, Novartis Pharmaceuticals Canada, Allergan, Nikon Canada, Bausch and Lomb Canada, and Bayer Canada, and been a paid advisory board member for Bayer Canada, Novartis Pharmaceuticals Canada, Allergan, and Alcon. M.A.K. has been a consultant for Novartis Pharmaceuticals Canada and Bayer Canada. P.J.K. has received research funding from Bayer Canada, Allergan, Novartis Pharmaceuticals Canada, and Roche Canada, and fellowship funding from Novartis Pharmaceuticals Canada and Bayer Canada; he has also participated in advisory boards for Novartis Pharmaceuticals Canada, Allergan, Roche Canada, and Bayer Canada, and scientific advisory boards for Novelty Nobility, and owns stock in Arctic Dx. C.X.Q. has been a consultant for Allergan, Bayer Canada, Novartis Pharmaceuticals Canada, and Bausch and Lomb. A.S. has been a consultant for and received research funding from Novartis Pharmaceuticals Canada and Bayer Canada. T.S. has been a consultant (study protocols for Peregrine and CANTREAT) for Novartis Pharmaceuticals Canada, has participated in advisory boards for Allergan, and is an investigator in active clinical trials for Roche Canada, Bayer Canada, Novartis Pharmaceuticals Canada, Allergan, and Chengdu Kanghoug Biotechnology. J.H.W. has received honoraria from Alcon, Novartis Pharmaceuticals Canada, and Bayer Canada, as well as a research grant from Novartis Pharmaceuticals Canada.

\section{Funding Sources}

All authors received an honorarium from Novartis Pharmaceuticals Canada. Novartis retained SNELL Medical Communication and provided financial assistance to organize the author conference, perform the literature research, and prepare a draft manuscript. The authors were provided complete editorial independence in the development of this article, and no Novartis personnel participated or were present during the author meeting.

\section{Author Contributions}

All authors contributed significantly to the outline, writing, and editorial review of this paper.

\section{References}

1 Pauleikhoff D. neovascular age-related macular degeneration: Natural History and TreatmentOutcomes. Retina.2005Dec;25(8):106584.

2 Brown DM, Kaiser PK, Michels M, Soubrane G, Heier JS, Kim RY, et al.; ANCHOR Study Group. Ranibizumab versus verteporfin for neovascular age-related macular degeneration. N Engl J Med. 2006 Oct;355(14):143244.

3 Rosenfeld PJ, Brown DM, Heier JS, Boyer DS, Kaiser PK, Chung CY, et al.; MARINA Study Group. Ranibizumab for neovascular age-related macular degeneration. N Engl J Med. 2006 Oct;355(14):1419-31.

4 Heier JS, Korobelnik JF, Brown DM, SchmidtErfurth U, Do DV, Midena E, et al. Intravit- real aflibercept for diabetic macular edema: 148-week results from the VISTA and VIVID studies. Ophthalmology. 2016 Nov;123(11): 2376-85.

5 Dugel PU, Koh A, Ogura Y, Jaffe GJ, SchmidtErfurth U, Brown DM, et al.; HAWK and HARRIER Study Investigators. HAWK and HARRIER: phase 3, multicenter, randomized, double-masked trials of brolucizumab for neovascular age related macular degeneration. Ophthalmology. 2020 Jan;127(1):7284.

6 Wykoff CC, Croft DE, Brown DM, Wang R, Payne JF, Clark L, et al.; TREX-AMD Study Group. Prospective trial of treat-and-extend versus monthly dosing for neovascular agerelated macular degeneration: TREX-AMD 1-year results. Ophthalmology. 2015 Dec; 122(12):2514-22.

7 Silva R, Berta A, Larsen M, Macfadden W, Feller C, Monés J; TREND Study Group. Treat-and-extend versus monthly regimen in neovascular age-related macular degeneration: results with ranibizumab from the TREND study. Ophthalmology. 2018 Jan; 125(1):57-65.

8 Kertes PJ, Galic IJ, Greve M, Williams RG, Rampakakis E, Scarino A, et al. Canadian Treat-and-Extend Analysis Trial with ranibizumab in patients with neovascular age-related macular disease: one-year results of the randomized Canadian Treat-and-Extend Analysis Trial with Ranibizumab Study. Ophthalmology. 2019 Jun;126(6):841-8. 
9 Martin DF, Maguire MG, Fine SL, Ying GS, Jaffe GJ, Grunwald JE, et al.; Comparison of Age-related Macular Degeneration Treatments Trials (CATT) Research Group. Ranibizumab and bevacizumab for treatment of neovascular age-related macular degeneration: two-year results. Ophthalmology. 2012 Jul;119(7):1388-98.

10 Ho AC, Busbee BG, Regillo CD, Wieland MR, Van Everen SA, Li Z, et al.; HARBOR Study Group. Twenty-four-month efficacy and safety of $0.5 \mathrm{mg}$ or $2.0 \mathrm{mg}$ ranibizumab in patients with subfoveal neovascular age-related macular degeneration. Ophthalmology. 2014 Nov;121(11):2181-92.

11 Holz FG, Amoaku W, Donate J, Guymer RH, Kellner U, Schlingemann RO, et al.; SUSTAIN Study Group. Safety and efficacy of a flexible dosing regimen of ranibizumab in neovascular age-related macular degeneration: the SUSTAIN study. Ophthalmology. 2011 Apr;118(4):663-71.

12 Cruess AF, Berger A, Colleaux K, Greve M, Harvey P, Kertes PJ, et al. Canadian expert consensus: optimal treatment of neovascular age-related macular degeneration. Can J Ophthalmol. 2012 Jun;47(3):227-35.

13 Freund KB, Zweifel SA, Engelbert M. Do we need a new classification for choroidal neovascularization in age-related macular degeneration? Retina. 2010 Oct;30(9):1333-49.

14 Jung JJ, Chen CY, Mrejen S, Gallego-Pinazo $\mathrm{R}, \mathrm{Xu} \mathrm{L}$, Marsiglia M, et al. The incidence of neovascular subtypes in newly diagnosed neovascular age-related macular degeneration. Am J Ophthalmol. 2014 Oct;158(4):76979.e2.

15 Zhao Z, Yang F, Gong Y, Yu S, Liu H, Wang $\mathrm{H}$, et al. The comparison of morphologic characteristics of type 1 and type 2 choroidal neovascularization in eyes with neovascular age-related macular degeneration using optical coherence tomography angiography. Ophthalmologica. 2019;242(3):178-86.

16 Arias-Barquet L, Monés J. Fluorescein Angiography [cited 14 Oct 2019]. Available from: http://www.amdbook.org/content/fluorescein-angiography- 0 .

17 Regillo CD, Blade KA, Custis PH, O'Connell SR. Evaluating persistent and recurrent choroidal neovascularization. The role of indocyanine green angiography. Ophthalmology. 1998 Oct;105(10):1821-6.

18 Desmettre T, Devoisselle JM, Mordon S. Fluorescence properties and metabolic features of indocyanine green (ICG) as related to angiography. Surv Ophthalmol. 2000;45(1):1527.

19 Lam WC, Choudhry N, Wong D. Polypoidal choroidal vasculopathy in Canada. Can J Ophthalmol. 2020 Jun;55(3):199-211.

20 Guyer DR, Yannuzzi LA, Ladas I, Slakter IS, Sorenson JA, Orlock D. Indocyanine greenguided laser photocoagulation of focal spots at the edge of plaques of choroidal neovascularization. Arch Ophthalmol. 1996 Jun; 114(6):693-7.
21 Guyer DR, Yannuzzi LA, Slakter JS, Sorenson JA, Hanutsaha P, Spaide RF, et al. Classification of choroidal neovascularization by digital indocyanine green videoangiography. Ophthalmology. 1996 Dec;103(12):2054-60.

22 Fernández M, Gil M, Gonzalez F, Gómez-Ulla F. Diagnostic Usefulness of Indocyanine Green Angiography (ICGA) in Age-related Macular Degeneration (AMD) [cited 2019 Oct 14]. Available from: http://www.amdbook.org/content/diagnostic-usefulness-indocyanine-green-angiography-icga-age-related-macular-degeneration-am.

23 Yannuzzi LA, Rohrer KT, Tindel LJ, Sobel RS, Costanza MA, Shields W, et al. Fluorescein angiography complication survey. Ophthalmology. 1986 May;93(5):611-7.

24 Meira J, Marques ML, Falcão-Reis F, Rebelo Gomes E, Carneiro Â. Immediate reactions to fluorescein and indocyanine green in retinal angiography: review of literature and proposal for patient's evaluation. Clin Ophthalmol. 2020 Jan;14:171-8.

25 Bonte CA, Ceuppens J, Leys AM. Hypotensive shock as a complication of infracyanine green injection. Retina. 1998;18(5):476-7.

26 Giani A, Esmaili DD, Luiselli C, Cigada M, Salvetti P, Miller JW, et al. Displayed reflectivity of choroidal neovascular membranes by optical coherence tomography correlates with presence of leakage by fluorescein angiography. Retina. 2011 May;31(5):942-8.

27 Giani A, Luiselli C, Esmaili DD, Salvetti P, Cigada M, Miller JW, et al. Spectral-domain optical coherence tomography as an indicator of fluorescein angiography leakage from choroidal neovascularization. Invest Ophthalmol Vis Sci. 2011 Jul;52(8):5579-86.

28 Coscas F, Coscas G, Souied E, Tick S, Soubrane G. Optical coherence tomography identification of occult choroidal neovascularization in age-related macular degeneration. Am J Ophthalmol. 2007 Oct;144(4):592-9.

29 Eter N, Spaide RF. Comparison of fluorescein angiography and optical coherence tomography for patients with choroidal neovascularization after photodynamic therapy. Retina. 2005 Sep;25(6):691-6.

30 Narita C, Wu Z, Rosenfeld PJ, Yang J, Lyu C, Caruso E, et al. Structural OCT signs suggestive of subclinical nonexudative macular neovascularization in eyes with large drusen. Ophthalmology. 2020 May;127(5):637-47.

31 Silva N, Marta A, Baptista P, Furtado MJ, Lume M. Optical coherence tomography findings (SD-OCT and OCTA) in early-stage type 3 neovascularization. Case Rep Ophthalmol. 2020 Aug;11(2):493-9.

32 Drexler W, Fujimoto JG. State-of-the-art retinal optical coherence tomography. Prog Retin Eye Res. 2008 Jan;27(1):45-88.

33 Fang PP, Domdei N, Herrmann P, SchmitzValckenberg S, Holz FG, Harmening WM, et al. Minimal optical coherence tomography Bscan density for reliable detection of intraretinal and subretinal fluid in macular diseases. Retina. 2019 Jan;39(1):150-6.
34 Michalewski J, Nawrocki J, Trebinska M, Michalewska Z. Spectral-domain optical coherence tomography features preceding new-onset neovascular membrane formation. Can J Ophthalmol. 2014 Aug;49(4):339-44.

35 Flores-Moreno I, Arias-Barquet L, Rubio-Caso MJ, Ruiz-Moreno JM, Duker JS, Caminal JM. En face swept-source optical coherence tomography in neovascular age-related macular degeneration. Br J Ophthalmol. 2015 Sep; 99(9):1260-7.

36 Koustenis A Jr, Harris A, Gross J, Januleviciene I, Shah A, Siesky B. Optical coherence tomography angiography: an overview of the technology and an assessment of applications for clinical research. Br J Ophthalmol. 2017 Jan;101(1):16-20.

37 Spaide RF, Klancnik JM Jr, Cooney MJ. Retinal vascular layers imaged by fluorescein angiography and optical coherence tomography angiography. JAMA Ophthalmol. 2015 Jan; 133(1):45-50

38 Rodríguez FJ, Staurenghi G, Gale R; Vision Academy Steering Committee. The role of OCT-A in retinal disease management. Graefes Arch Clin Exp Ophthalmol. 2018 Nov; 256(11):2019-26.

39 Spaide RF, Fujimoto JG, Waheed NK, Sadda SR, Staurenghi G. Optical coherence tomography angiography. Prog Retin Eye Res. 2018 May;64:1-55.

40 Borrelli E, Sadda SR, Uji A, Querques G. Pearls and pitfalls of optical coherence tomography angiography imaging: a review. Ophthalmol Ther. 2019 Jun;8(2):215-26.

41 Borrelli E, Sarraf D, Freund KB, Sadda SR. OCT angiography and evaluation of the choroid and choroidal vascular disorders. Prog Retin Eye Res. 2018 Nov;67:30-55.

42 Coscas GJ, Lupidi M, Coscas F, Cagini C, Souied EH. Optical coherence tomography angiography versus traditional multimodal imaging in assessing the activity of exudative age-related macular degeneration: a new diagnostic challenge. Retina. 2015 Nov;35(11): 2219-28

43 Heiferman MJ, Fawzi AA. Progression of subclinical choroidal neovascularization in agerelated macular degeneration. PLoS One. 2019 Jun;14(6):e0217805.

44 Soomro T, Talks J; Medscape. The use of optical coherence tomography angiography for detecting choroidal neovascularization, compared to standard multimodal imaging. Eye (Lond). 2018 Apr;32(4):661-72.

45 Yung M, Klufas MA, Sarraf D. Clinical applications of fundus autofluorescence in retinal disease. Int J Retina Vitreous. 2016 Apr; 2(1):12.

46 Mrejen S, Sarraf D, Mukkamala SK, Freund $\mathrm{KB}$. Multimodal imaging of pigment epithelial detachment: a guide to evaluation. Retina. 2013 Oct;33(9):1735-62.

47 Mimoun G, Soubrane G, Coscas G. Macular drusen. J Fr Ophtalmol. 1990;13(10):511-30. French. 
48 Pumariega NM, Smith RT, Sohrab MA, Letien V, Souied EH. A prospective study of reticular macular disease. Ophthalmology. 2011 Aug;118(8):1619-25.

49 Camacho N, Barteselli G, Nezgoda JT, ElEmam S, Cheng L, Bartsch DU, et al. Significance of the hyperautofluorescent ring associated with choroidal neovascularisation in eyes undergoing anti-VEGF therapy for wet agerelated macular degeneration. Br J Ophthalmol. 2015 Sep;99(9):1277-83.

50 Schmidt-Erfurth U, Waldstein SM. A paradigm shift in imaging biomarkers in neovascular age-related macular degeneration. Prog Retin Eye Res. 2016 Jan;50:1-24.

51 Jaffe GJ, Martin DF, Toth CA, Daniel E, Maguire MG, Ying GS, et al.; Comparison of Age-related Macular Degeneration Treatments Trials Research Group. Macular morphology and visual acuity in the comparison of age-related macular degeneration treatments trials. Ophthalmology. 2013 Sep; 120(9):1860-70.

52 Simader C, Ritter M, Bolz M, Deák GG, MayrSponer U, Golbaz I, et al. Morphologic parameters relevant for visual outcome during anti-angiogenic therapy of neovascular agerelated macular degeneration. Ophthalmology. 2014 Jun;121(6):1237-45.

53 Waldstein SM, Simader C, Staurenghi G, Chong NV, Mitchell P, Jaffe GJ, et al. Morphology and visual acuity in aflibercept and ranibizumab therapy for neovascular age-related macular degeneration in the VIEW trials. Ophthalmology. 2016 Jul;123(7):1521-9.

54 Lai TT, Hsieh YT, Yang CM, Ho TC, Yang $\mathrm{CH}$. Biomarkers of optical coherence tomography in evaluating the treatment outcomes of neovascular age-related macular degeneration: a real-world study. Sci Rep. 2019 Jan; 9(1):529.

55 Gianniou C, Dirani A, Jang L, Mantel I. Refractory intraretinal or subretinal fluid in neovascular age-related macular degeneration treated with intravitreal ranibizumab: functional and structural outcome. Retina. 2015 Jun;35(6):1195-201.

56 Schmidt-Erfurth U, Waldstein SM, Deak GG, Kundi M, Simader C. Pigment epithelial detachment followed by retinal cystoid degeneration leads to vision loss in treatment of neovascular age-related macular degeneration. Ophthalmology. 2015 Apr;122(4):82232.

57 Sadda SR, Tuomi LL, Ding B, Fung AE, Hopkins JJ. Macular atrophy in the HARBOR Study for neovascular age-related macular degeneration. Ophthalmology. 2018 Jun;125(6): $878-86$.

58 Sato T, Suzuki M, Ooto S, Spaide RF. Multimodal imaging findings and multimodal vision testing in neovascular age-related macular degeneration. Retina. 2015 Jul;35(7):1292302.

59 Wickremasinghe SS, Janakan V, Sandhu SS, Amirul-Islam FM, Abedi F, Guymer RH. Implication of recurrent or retained fluid on op- tical coherence tomography for visual acuity during active treatment of neovascular agerelated macular degeneration with a treat and extend protocol. Retina. 2016 Jul;36(7):13319.

60 Golbaz I, Ahlers C, Stock G, Schütze C, Schriefl S, Schlanitz F, et al. Quantification of the therapeutic response of intraretinal, subretinal, and subpigment epithelial compartments in exudative AMD during anti-VEGF therapy. Invest Ophthalmol Vis Sci. 2011 Mar;52(3):1599-605.

61 Guymer RH, Markey CM, McAllister IL, Gillies MC, Hunyor AP, Arnold JJ, et al.; FLUID Investigators. FLUID Investigators. Tolerating subretinal fluid in neovascular age-related macular degeneration treated with ranibizumab using a treat-and-extend regimen: FLUID Study 24-Month Results. Ophthalmology. 2019 May;126(5):723-34.

62 Penha FM, Gregori G, Garcia Filho CA, Yehoshua Z, Feuer WJ, Rosenfeld PJ. Quantitative changes in retinal pigment epithelial detachments as a predictor for retreatment with anti-VEGF therapy. Retina. 2013 Mar; 33(3):459-66

63 Sasaki M, Kato Y, Fujinami K, Hirakata T, Tsunoda K, Watanabe $\mathrm{K}$, et al. Advanced quantitative analysis of the sub-retinal pigment epithelial space in recurrent neovascular age-related macular degeneration. PLoS One. 2017 Nov;12(11):e0186955.

64 Klimscha S, Waldstein SM, Schlegl T, Bogunovic $\mathrm{H}$, Sadeghipour A, Philip AM, et al. Spatial correspondence between intraretinal fluid, subretinal fluid, and pigment epithelial detachment in neovascular age-related macular degeneration. Invest Ophthalmol Vis Sci. 2017 Aug;58(10):4039-48.

65 Sarraf D, Joseph A, Rahimy E. Retinal pigment epithelial tears in the era of intravitreal pharmacotherapy: risk factors, pathogenesis, prognosis and treatment (an American Ophthalmological Society thesis). Trans Am Ophthalmol Soc. 2014 Jul;112:142-59.

66 Dervenis N, Younis S. Macular morphology and response to ranibizumab treatment in $\mathrm{pa}$ tients with wet age-related macular degeneration. Clin Ophthalmol. 2016 Jun;10:1117-22.

67 Jhaveri CD, Dugel PU, Wykoff CC, et al. 96week visual and expanded anatomical outcomes of brolucizumab versus aflibercept in patients with nAMD: Results from HAWK and HARRIER. Presented at the ASRS 37th Annual Scientific Meeting. July 27, 2019; Chicago, IL.

68 Evans RN, Chakravarthy U, Reeves B. Associations between variation in retinal thickness and visual function. Presented at the ARVO Annual Meeting. April 28-May 2, 2019: Vancouver, BC. Poster 3456.

69 Jaffe GJ, Ying GS, Toth CA, Daniel E, Grunwald JE, Martin DF, et al.; Comparison of Age-related Macular Degeneration Treatments Trials Research Group. Macular morphology and visual acuity in year five of the Comparison of Age-related Macular Degen- eration Treatments Trials. Ophthalmology. 2019 Feb;126(2):252-60.

70 Waldstein SM, Wright J, Warburton J, Margaron P, Simader C, Schmidt-Erfurth U. Predictive value of retinal morphology for visual acuity outcomes of different ranibizumab treatment regimens for neovascular AMD. Ophthalmology. 2016 Jan;123(1):60-9.

71 Agarwal A, Rhoades WR, Hanout M, Soliman MK, Sarwar S, Sadiq MA, et al. Management of neovascular age-related macular degeneration: current state-of-the-art care for optimizing visual outcomes and therapies in development. Clin Ophthalmol. 2015 Jun;9:1001-15.

72 Schmidt-Erfurth U, Chong V, Loewenstein A, Larsen M, Souied E, Schlingemann R, et al.; European Society of Retina Specialists. Guidelines for the management of neovascular agerelated macular degeneration by the European Society of Retina Specialists (EURETINA). Br J Ophthalmol. 2014 Sep;98(9):1144-67.

73 Chakravarthy U, Williams M; AMD Guidelines Group. The Royal College of Ophthalmologists Guidelines on AMD: executive Summary. Eye(Lond).2013 Dec;27(12):1429_ 31.

74 Ashraf M, Souka A, Adelman RA. Age-related macular degeneration: using morphological predictors to modify current treatment protocols. Acta Ophthalmol. 2018 Mar;96(2):12033.

75 Qian T, Li X, Zhao M, Xu X. Polypoidal choroidal vasculopathy treatment options: A meta-analysis. Eur J Clin Invest. 2018 Jan;48(1): e12840.

76 Koh A, Lee WK, Chen LJ, Chen SJ, Hashad Y, Kim H, et al. EVEREST study: efficacy and safety of verteporfin photodynamic therapy in combination with ranibizumab or alone versus ranibizumab monotherapy in patients with symptomatic macular polypoidal choroidal vasculopathy. Retina. 2012 Sep;32(8): 1453-64.

77 Wong TY, Ogura Y, Lee WK, Iida T, Chen SJ, Mitchell P, et al.; PLANET Investigators. PLANET Investigators. Efficacy and safety of intravitreal aflibercept for polypoidal choroidal vasculopathy: two-year results of the Aflibercept in Polypoidal Choroidal Vasculopathy Study. Am J Ophthalmol. 2019 Aug;204:80-9.

78 Kramann CA, Schöpfer K, Lorenz K, Zwiener I, Stoffelns BM, Pfeiffer N. Intravitreal ranibizumab treatment of retinal angiomatous proliferation. Acta Ophthalmol. 2012 Aug;90(5): 487-91.

79 Montero JA, Fernandez MI, Gomez-Ulla F, Ruiz-Moreno JM. Efficacy of intravitreal bevacizumab to treat retinal angiomatous proliferation stage II and III. Eur J Ophthalmol. 2009 May-Jun;19(3):448-51

80 Seidel G, Werner C, Weger M, Steinbrugger I, Haas A. Combination treatment of photodynamic therapy with verteporfin and intravitreal ranibizumab in patients with retinal angiomatous proliferation. Acta Ophthalmol. 2013 Sep;91(6):e482-5. 
81 Freund KB, Klais CM, Eandi CM, Ober MD, Goldberg DE, Sorenson JA, et al. Sequenced combined intravitreal triamcinolone and indocyanine green angiography-guided photodynamic therapy for retinal angiomatous proliferation. Arch Ophthalmol.2006 Apr;124(4): 487-92.

82 Vardarinos A, Gupta N, Janjua R, Iron A, Empeslidis T, Tsaousis KT. 24-month clinical outcomes of a treat-and-extend regimen with ranibizumab for wet age-related macular degeneration in a real-life setting. BMC Ophthalmol. 2017 Apr;17(1):58.

83 Schmidt-Erfurth U, Kaiser PK, Korobelnik JF, Brown DM, Chong V, Nguyen QD, et al. Intravitreal aflibercept injection for neovascular age-related macular degeneration: ninety-sixweek results of the VIEW studies. Ophthalmology. 2014 Jan;121(1):193-201.

84 Sharma A, Kumar N, Kuppermann BD, Loewenstein A, Bandello F. Brolucizumab: is extended VEGF suppression on the horizon? Eye (Lond). 2020 Mar;34(3):424-6.

85 Holz FG, Dugel PU, Weissgerber G, Hamilton R, Silva R, Bandello F, et al. Single-chain antibody fragment VEGF inhibitor RTH258 for neovascular age-related macular degeneration. Ophthalmology. 2016 May;123(5): 1080-9.

86 Novartis AG. Beovu ${ }^{\circledR}$ (brolucizumab) global use and safety information for healthcare professionals [cited 5 January, 2021]. Available from: https://www.brolucizumab.info.

87 Monés J, Srivastava SK, Jaffe GJ, et al. Risk of inflammation, retinal vasculitis, and retinal occlusion - related events with brolucizumab: post hoc review of HAWK and HARRIER. Ophthalmology. 2020, Online ahead of print.

88 Hussain RM, Neiweem AE, Kansara V, Harris A, Ciulla TA. Tie-2/Angiopoietin pathway modulation as a therapeutic strategy for retinal disease. Expert Opin Investig Drugs. 2019 Oct;28(10):861-9.

89 Chakravarthy U, Bailey C, Brown D, Campochiaro P, Chittum M, Csaky K, et al. Phase 1 trial of anti-vascular endothelial growth factor/antiangiopoietin 2 bispecific antibody RG7716 for neovascular age-related macular degeneration. Ophthalmol Retina. 2017;1(6): $474-85$.
90 Rangasamy S, Srinivasan R, Maestas J, McGuire PG, Das A. A potential role for angiopoietin 2 in the regulation of the blood-retinal barrier in diabetic retinopathy. Invest Ophthalmol Vis Sci. 2011 Jun;52(6):3784-91.

91 Roche. Roche's faricimab meets primary endpoint in two global phase III studies and shows potential to extend time between treatments up to 16 weeks for people with neovascular age-related macular degeneration. JAMA Ophthalmol. January 25, 2021 [cited June 1, 2021. Available from: https://www.roche. com/media/releases/med-cor-2021-01-25. htm.

92 Roche. Phase III data show Roche's Port Delivery System with ranibizumab enabled over $98 \%$ of patients to go six months between treatments for neovascular age-related macular degeneration. July 22, 2020 [cited 5 January, 2021]. Available from: https://www.roche.com/media/releases/med-cor-2020-07-22b.htm.

93 Extension Study for the Port Delivery System with Ranibizumab (Portal) [cited 5 January, 2021]. Available from: https://clinicaltrials. gov/ct2/show/NCT03683251. 